\title{
Densidade e tamanho populacional de mamíferos cinegéticos em duas Unidades de Conservação do Estado do Rio de Janeiro, Brasil
}

\author{
Roberta M. de Araújo, Maíra B. de Souza \& Carlos R. Ruiz-Miranda
}

\begin{abstract}
Laboratório de Ciências Ambientais, Centro de Biociências e Biotecnologia, Universidade Estadual do Norte Fluminense. Av. Alberto Lamego, 2000 - Horto. Campos dos Goytacazes, 28013-600 Rio de Janeiro, RJ. (roby_araujo@hotmail.com; mairabs02@gmail.com; cruiz@uenf.br).
\end{abstract}

\begin{abstract}
Density and population size of game mammals in two Conservation Units of the State of Rio de Janeiro, Brazil. The Atlantic Rain Forest even though suffering intense devastation, shelters 261 species of mammals, 73 endemic. Large mammals were among the most vulnerable to hunting, loss of habitat, and wildlife trade. In the State of Rio de Janeiro there are only two Federal Biological Reserves of lowland Atlantic Rain Forest, the Poço das Antas Biological Reserve and the União Biological Reserve. The purpose of this study was to assess the presence and putative influence of illegal hunting on the mammals in these two Conservation Units. Surveys were conducted using a line-transect method; 375 kilometers were covered during the period between December 2003 and January 2005. The data for the population density estimate was analyzed using the program DISTANCE 5.0. Twelve species were confirmed through visual encounters during the surveys, whereas are regularly hunted in the region studied. The species that presented higher density were brown capuchin monkey (Cebus nigritus Erxleben, 1777), howler monkey (Alouatta guariba Lacépède, 1799), ninebanded long-nosed armadillo (Dasypus novemcintus Linnaeus, 1758), and the seven-banded long-nosed armadillo (Dasypus septemcinctus Linnaeus, 1758). The most rare or absent species were the brazilian tapir (Tapirus terrestris Brünnich, 1771), the red brocket deer (Mazama americana Rafinesque, 1817) and the white-lipped peccary (Tayassu pecari Link, 1795). In these two study areas both direct and indirect evidence of illegal hunting was observed, indicating that hunting is a common practice inside these Biological Reserves. The long-term survival of the hunted species is questionable, because the remaining populations living in fragments that are small and isolated, making them more susceptible to extinction even under low hunting pressure.
\end{abstract}

KEYWORDS. Mammals, hunting, forest fragmentation, game species, poaching.

RESUMO. A Mata Atlântica, apesar de ainda sofrer uma intensa devastação, abriga 261 espécies de mamíferos, sendo 73 endêmicos. Mamíferos de grande porte estão entre os mais vulneráveis à caça, perda de habitat e tráfico de animais. No Estado do Rio de Janeiro existem somente duas Reservas Biológicas de Mata Atlântica de baixada, a Reserva Biológica de Poço das Antas e a Reserva Biológica União. O presente estudo teve como objetivo avaliar a influência da prática da caça ilegal sobre a fauna de mamíferos nestas duas Unidades de Conservação. O levantamento populacional foi realizado utilizando o método de transecção linear e 375 quilômetros foram percorridos durante o período de dezembro de 2003 a janeiro de 2005. Os dados de estimativa de densidade populacional foram analisados no programa DISTANCE 5.0. Através de encontros visuais foram confirmadas 12 espécies durante o levantamento, sendo estas regularmente caçadas na região. As espécies que apresentaram maior densidade nas duas Unidades de Conservação foram o macaco-prego (Cebus nigritus Erxleben, 1777), o bugio (Alouatta guariba Lacépède, 1799), tatu-galinha (Dasypus novemcintus Linnaeus, 1758) e o tatu (Dasypus septemcinctus Linnaeus, 1758). As espécies mais raras ou ausentes foram a anta (Tapirus terrestris Brünnich, 1771), o veado (Mazama americana Rafinesque, 1817) e o queixada (Tayassu pecari Link, 1795). Evidências diretas e indiretas da ação da caça ilegal foram observadas nas duas áreas de estudo, indicando que a caça é uma prática comum nessas Reservas Biológicas. A sobrevivência a longo prazo dessas espécies é questionável, já que as populações remanescentes em fragmentos são pequenas e isoladas, o que as tornam muito susceptível à extinção mesmo sob uma baixa pressão de caça.

PALAVRAS-CHAVE. Mamíferos, caça, fragmentação florestal, Mata Atlântica, Unidades de Conservação.

A Mata Atlântica cobria originalmente uma área em torno de 1.100,00 quilômetros quadrados ao longo da costa brasileira, da região Nordeste até a Sul. O Estado do Rio de Janeiro, de acordo com projeções históricas, possuía 97\% da área coberta por esse bioma (SOS MATA AtLÂNTICA/INPE, 1993; MitTERMEIER et al., 1998). Com uma população atual superior a 13 milhões de habitantes, a área de cobertura florestal do Estado está reduzida a menos de $17 \%$ em relação à existente originalmente (TANIZAKI-FonseCA \& Moulton, 2000; Rocha et al., 2003).

Apesar de ainda ocorrer intensa devastação, a Mata Atlântica abriga 261 espécies de mamíferos, sendo 73 endêmicos (MYers et al., 2000). Mamíferos de grande porte estão entre os mais vulneráveis à caça, perda de habitat e tráfico de animais (LANE, 1990; REDFORD, 1992; Chiarello, 2000b; Cullen et al., 2000). Contudo, poucos são os trabalhos que fornecem dados sobre a abundância e a densidade populacional de mamíferos que ainda persistem em fragmentos de Mata Atlântica (ChIARELlo, 2000a; Chiarello, 2000b; Chiarello \& Melo, 2001).

Comunidades de mamíferos e aves frugívoras sofrem com a pressão da caça ilegal que, continua sendo praticada de maneira esportiva ou recreativa e até mesmo profissional na maioria das áreas de Mata Atlântica (GALETTI et al., 1997; Chiarello, 2000b). Peres (2000) afirmou que a caça e a fragmentação florestal afetam negativamente a comunidade de vertebrados em fragmentos amazônicos, pois reduzem seu tamanho populacional, biomassa e diversidade, além de facilitar o acesso de caçadores à mata (CHIARELlO 1999; Chiarello 2000b; Cullen et al., 2000).

Apenas 2\% das áreas de Mata Atlântica encontramse sob proteção legal (MYers et al., 2000). As Unidades de Conservação estabelecidas tiveram como objetivo principal a conservação e proteção da biodiversidade, porém a caça e a extração ilegal de recursos naturais ainda persistem nestas áreas (Olmos et al., 2002). No Estado 
do Rio de Janeiro, as Reservas Biológicas de Poço das Antas e União estão submetidas a interferências antrópicas. O estabelecimento de empreendimentos como ferrovia, rodovia, linhas de transmissão de energia elétrica e barragem fazem com que estas Unidades de Conservação se tornem fragmentadas e facilite a entrada de caçadores provenientes do entorno.

As Reservas Biológicas de Poço das Antas e União representam um dos últimos remanescentes de Mata Atlântica de Baixada Costeira do Estado do Rio de Janeiro e abrigam diversas espécies de aves (Pipile jacutinga Spix, 1825, Tinamus solitarius Vieillot, 1819) e mamíferos (Alouatta guariba Lacépède, 1799, Cebus nigritus Erxleben, 1777, Pecari tajacu Linnaeus, 1758, Agouti paca Linnaeus, 1766) (SEMA, 2001; BRITO et al., 2004), entre outras. As duas Unidades de Conservação foram criadas com o objetivo de assegurar a proteção e a recuperação de remanescentes de Floresta Atlântica e das formações associadas, assim como de populações de micos-leões-dourados (Leontopithecus rosalia Linnaeus, 1766), primata endêmico do Estado do Rio de Janeiro (SEMA, 2001)

Avaliar as condições populacionais da fauna localizada em áreas que sofrem interferência antrópica é fundamental para assegurarmos a proteção efetiva dentro de Unidades de Conservação. Assim, este estudo tem como objetivos estimar a abundância, densidade e tamanho populacional de mamíferos cinegéticos e analisar evidências da caça ilegal em duas Unidades de Conservação do Estado do Rio de Janeiro.

\section{MATERIAL E MÉTODOS}

Área de estudo. Os dados de levantamento populacional foram coletados em dois fragmentos de Mata Atlântica de Baixada do Estado do Rio de Janeiro, Reserva Biológica de Poço das Antas (REBIO de Poço

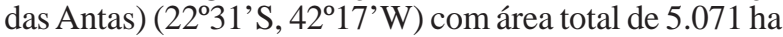
e Reserva Biológica União (REBIO União) (22²7' S, $42^{\circ} 02^{\prime} \mathrm{W}$ ) com 2.927 ha (Fig. 1). Na região predomina $\mathrm{o}$ clima tropical úmido, com a temperatura média anual de $24,6^{\circ} \mathrm{C}$ e precipitação média anual entre 1.100 a $2.121 \mathrm{~mm}$. Existe uma sazonalidade definida, com a estação seca compreendida entre junho e agosto e estação chuvosa entre setembro e maio (SEMA, 2001).

Os dois fragmentos estudados apresentam semelhanças na composição florística, topografia e condições climáticas (AMLD, 2003). No entanto, a REBIO União apresenta uma comunidade arbórea rica e com maior biomassa do que a REBIO de Poço das Antas (AMLD, 2003). Sinais de um passado de distúrbios antropogênicos existem nos fragmentos, incluindo caça, corte seletivo de madeira, plantações de eucalipto, passagem de rodovias e estradas (Tab. I).
O nível de distúrbio da REBIO de Poço das Antas foi considerado alto principalmente pela existência da Barragem de Juturnaíba que afeta consideravelmente esta Unidade de Conservação. A barragem alagou terras à montante e promoveu uma forte drenagem à juzante, $\mathrm{o}$ que ocasionou a modificação de parte do ecossistema, que era anteriormente de matas inundáveis (Tab. I) (PIRES \& FERnANDEZ, 1999; PIRES et al., 2002).

Coleta de dados. Para estimar a abundância, densidade e tamanho populacional de mamíferos cinegéticos nas duas Unidades de Conservação, foi realizado um levantamento populacional utilizando o método de transecção linear (BUCKLAND et al., 1993). Foram consideradas como cinegéticas todas as espécies de mamíferos diurnos tradicionalmente caçados na Mata Atlântica como: anta (Tapirus terrestris Brünnich, 1771), veado (Mazama americana Rafinesque, 1817), queixada (Tayassu pecari Link, 1795), cateto (P. tajacu), tatugalinha (Dasypus novemcinctus Linnaeus, 1758), tatu (Dasypus septemcinctus Linnaeus, 1758), capivara (Hydrochaeris hydrochaeris, Brünnich, 1771) e cutia
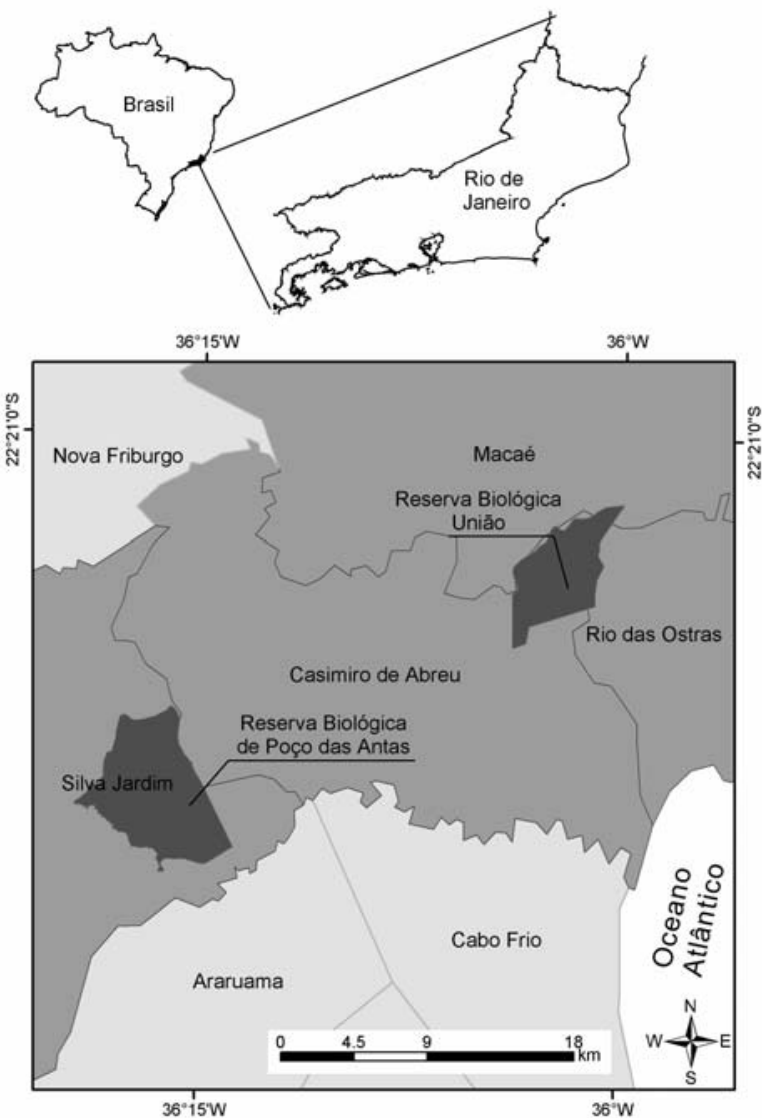

Fig. 1. Localização da Reserva Biológica de Poço das Antas e Reserva Biológica União, Rio de Janeiro.

Tabela I. Características ambientais e estado de conservação das Reservas Biológicas de Poço das Antas e União: área de floresta, grau de isolamento, nível de distúrbio, estado de conservação e tipo florestal predominante (1, Área de floresta estimada com base de mapas existentes; 2, Distúrbio florestal causado por fogo e/ou corte seletivo de madeira; 3, Composição florística e comunidade arbórea; 4, Tipo florestal que cobre mais de $50 \%$ da área da reserva).

\begin{tabular}{|c|c|c|c|c|c|c|}
\hline Área de estudo & Área (ha) & Área de floresta $(\mathrm{ha})^{1}$ & $\begin{array}{c}\text { Grau de } \\
\text { isolamento }\end{array}$ & $\begin{array}{l}\text { Nível de } \\
\text { distúrbio }^{2}\end{array}$ & $\begin{array}{c}\text { Estado de } \\
\text { conservação }\end{array}$ & $\begin{array}{l}\text { Tipo florestal } \\
\text { predominante }\end{array}$ \\
\hline Poço das Antas & 5.071 & $3.215(63,4 \%)$ & Alto & Alto & Moderado & Secundária \\
\hline União & 2.927 & $2.145(73,3 \%)$ & Conectado & Moderado & $\begin{array}{c}\text { Moderado - } \\
\text { Bem Conservado }\end{array}$ & Secundária \\
\hline
\end{tabular}


(Dasyprocta leporina, Illger, 1811). Espécies de primatas de grande porte como macaco-prego (C. nigritus) e bugio (A. guariba) também são vítimas de caçadores localmente (Cullen \& Valladares-Padua, 1997; Chiarello, 2000b; Cullen et al., 2000). O mico-leão-dourado (L. rosalia) não é alvo de caçadores atualmente devido ao trabalho de conservação realizado pela Associação Mico-LeãoDourado, ONG atuante há mais de 15 anos. Assim, este primata não foi considerado espécie cinegética neste estudo.

Observações e caminhadas foram feitas amostrando as diversas variações da vegetação nos locais e algumas trilhas já existentes foram usadas. O levantamento foi realizado das 5:30 às 17:30 horas, porque a probabilidade de avistamento de mamíferos diurnos aumenta durante estes horários (FERRARI, 2002). Caminhadas em trilhas, de comprimento determinado previamente, foram feitas em velocidade média constante de aproximadamente $1 \mathrm{~km} / \mathrm{h}$. $\mathrm{O}$ mesmo esforço amostral foi realizado entre as duas áreas de estudo.

Na REBIO de Poço das Antas foram utilizadas 10 trilhas que variaram de 600 a 2.250 metros de comprimento e na REBIO União 11 trilhas de 600 a 2.700 metros (Tab. II). Devido à baixa densidade de algumas espécies existentes nas áreas, um grande esforço amostral foi feito para atingir o número suficiente de avistamentos. Assim, foram percorridos 375 quilômetros durante o período de dezembro de 2003 a janeiro de 2005 para a coleta de dados no campo, sendo 194,8 quilômetros na REBIO de Poço das Antas e 180,2 quilômetros na REBIO União.

Dois observadores procuraram as espécies desejadas tanto à esquerda quanto à direita das trilhas. No momento em que um indivíduo ou o grupo foi visualizado os seguintes dados foram registrados: espécie, tamanho e composição do grupo, distância perpendicular do primeiro animal avistado até a trilha, distância entre o observador e ângulo de avistamento

Tabela II. Descrição das trilhas percorridas na Reserva Biológica de Poço das Antas e na Reserva Biológica União, RJ.

\begin{tabular}{|c|c|c|c|}
\hline Reserva Biológica & Trilhas & $\begin{array}{l}\text { Comprimento } \\
(\mathrm{Km})\end{array}$ & $\begin{array}{c}\mathrm{Km} \\
\text { percorrido }\end{array}$ \\
\hline \multirow[t]{10}{*}{ Poço das Antas } & Rodolfo Norte & 1,00 & 17,00 \\
\hline & Rodolfo Sul & 0,75 & 15,75 \\
\hline & Rio Preto & 2,25 & 25,20 \\
\hline & Casa dos Morcegos & 1,20 & 12,00 \\
\hline & Pelonha & 1,20 & 12,00 \\
\hline & Barragem & 2,00 & 22,00 \\
\hline & Aristides & 0,75 & 23,25 \\
\hline & A. desativada & 0,60 & 16,80 \\
\hline & Caju & 0,80 & 25,60 \\
\hline & Portuense & 1,20 & 25,20 \\
\hline \multirow[t]{11}{*}{ União } & Interpretativa & 2,70 & 35,10 \\
\hline & Heraldo & 0,85 & 17,02 \\
\hline & Três Pontes & 2,70 & 35,10 \\
\hline & LB & 0,95 & 13,30 \\
\hline & LB3 & 0,90 & 13,50 \\
\hline & Tanque & 0,60 & 10,08 \\
\hline & Chuchu & 0,80 & 12,00 \\
\hline & Grota & 0,75 & 11,25 \\
\hline & Cardoso & 0,60 & 11,40 \\
\hline & Fogueira & 0,67 & 10,05 \\
\hline & Figueira & 0,60 & 11,40 \\
\hline Total & & & 375 \\
\hline
\end{tabular}

(Cullen \& Valladares-Padua, 1997; Cullen et al., 2003). A distância perpendicular do animal até o transecto foi calculada posteriormente através do uso do ângulo e da distância de avistamento. Trena e bússola foram utilizadas para medir a distância de avistamento e o ângulo, respectivamente.

Além do levantamento, dados relativos a vocalizações, fezes e pegadas foram usados como evidências da presença de espécies nas duas Unidades de Conservação. Entrevistas com funcionários e pesquisadores foram realizadas para confirmar a presença de algumas espécies não avistadas durante o levantamento.

A densidade populacional foi estimada através do número total de indivíduos observados ao longo de cada trilha através do programa DISTANCE 5.0 (modelo "Half Normal", função Cosseno, Seqüencial) (BuCKLAND et al., 2001; Cullen et al., 2003).

O programa DISTANCE 5.0 utiliza as distâncias perpendiculares (animal-trilha) para estimar a largura efetiva da área amostrada (chamado ESW ou effective strip width), e assim, modelar a função de detecção de um animal numa dada distância da trilha (BUCKLAND et al., 2001; Cullen et al., 2003). Métodos de distância permitem obter estimativas de densidade populacional acuradas, mesmo que parte dos objetos de estudo não seja detectada (BUCKLAND et al., 2001).

A densidade foi calculada para cada espécie através da fórmula $\mathrm{D}=\mathrm{N} /(2 \cdot \mathrm{ESW} \cdot \mathrm{L})$, onde $\mathrm{D}=$ densidade (indivíduos $/ \mathrm{Km}^{2}$ ); $\mathrm{N}=$ número total de encontros; $\mathrm{ESW}=$ largura efetiva da área amostrada em quilômetros, calculada pelo programa DISTANCE; $\mathrm{L}=$ quilometragem total percorrida. A abundância foi estimada para cada espécie avistada como o total de avistamentos realizados a cada $10 \mathrm{Km}$ percorridos e o tamanho populacional das espécies que apresentaram maior abundância foi calculado multiplicando a densidade pela área de mata presente nos fragmentos estudados.

Dados sobre evidências diretas (encontro com caçadores, encontro com cães e tiros escutados) e indiretas (armadilhas, latidos de cães, ceva e poleiros) resultado da prática ilegal de caça nas duas Unidades de Conservação, foram coletados.

\section{RESULTADOS}

Evidências de caça ilegal foram encontradas nas duas áreas de estudo (Tab. III), sendo o encontro com caçadores e o avistamento de poleiros os indícios mais

Tabela III. Evidências diretas e indiretas da presença da prática ilegal da caça na área de estudo $\left(\chi^{2}, 5,76 ;\right.$ g.l., $1 ;$ p, 3,84).

\begin{tabular}{llcc}
\hline & & $\begin{array}{c}\text { Reserva } \\
\text { Biológica } \\
\text { de Poço } \\
\text { das Antas }\end{array}$ & $\begin{array}{c}\text { Reserva } \\
\text { Biológica } \\
\text { União }\end{array}$ \\
\hline Evidência & Encontro com caçadores & 2 & 6 \\
direta de caça & Encontro com cães & 1 & 2 \\
& Tiros escutados & 2 & 9 \\
Evidência & Armadilhas & - & - \\
indireta de caça & Latidos de cães & 2 & 2 \\
& Poleiros encontrados & 2 & 3 \\
& Ceva & 1 & 2 \\
\hline Total & & 10 & 24 \\
\hline
\end{tabular}


abundantes. A REBIO União apresentou maior número de vestígios de caça. Contudo, o total de evidências diretas e indiretas de caça entre as duas Unidades de Conservação não apresentaram diferença significativa $\left(\chi^{2}=5,76\right.$; g.l. $=1$; $\mathrm{p}=3,84)$.

Foram obtidos 64 encontros visuais com primatas tradicionalmente caçados nas duas Unidades de Conservação. Cebus nigritus foi avistado com maior freqüência na REBIO de Poço das Antas (22 encontros), enquanto na REBIO União esta espécie foi menos visualizada (8 encontros). Alouatta guariba foi visualizado com maior freqüência na REBIO União (20 encontros) e 14 encontros foram realizados na REBIO de Poço das Antas (Tab. IV).

A presença do $M$. americana foi confirmada por meio de pegadas avistadas somente na REBIO de Poço das Antas. Nenhum avistamento de $H$. hydrochaeris ocorreu na REBIO União. Pecari tajacu foi a única espécie de porco-do-mato encontrada nas duas Unidades de Conservação. Pesquisadores que trabalham há vários anos nos locais relataram que desde que iniciaram as suas pesquisas há mais de 15 anos nunca identificaram a presença de T. terrestris (Tab. IV).

Nenhuma espécie cuja densidade populacional foi estimada apresentou coeficiente de variação acima de $20 \%$ (Tab. V), valor este máximo recomendado para obter estimativa de densidade acurada (BUCKLAND et al., 1993).

Os mamíferos que apresentaram maior estimativa de densidade populacional e abundância nas duas

Tabela IV. Número de encontros durante o levantamento e evidências de mamíferos cinegéticos na área de estudo (pe, pegadas; vi, espécie vista fora do levantamento; -, espécie rara ou ausente).

\begin{tabular}{lcc}
\hline Espécies & $\begin{array}{c}\text { Reserva Biológica } \\
\text { de Poço das Antas }\end{array}$ & $\begin{array}{c}\text { Reserva Biológica } \\
\text { União }\end{array}$ \\
\hline Alouatta guariba & 14 & 20 \\
Cebus nigritus & 22 & 8 \\
Mazama americana & $\mathrm{pe}$ & - \\
Tapirus terrestris & - & - \\
Pecari tajacu & 6 & 6 \\
Tayassu pecari & - & - \\
Dasyprocta leporina & 6 & 4 \\
Hydrochaeris hydrochaeris & $\mathrm{vi}$ & $\mathrm{pe}$ \\
Dasypus novemcinctus & 25 & 15 \\
Dasypus septemcinctus & 5 & 7 \\
\hline Total & 83 & 64
\end{tabular}

Unidades de Conservação foram A. guariba, C. nigritus e D. novemcinctus. A densidade populacional estimada para A. guariba nas duas áreas de estudo foi similar, com 44,1 ind/ $\mathrm{Km}^{2}$ na REBIO de Poço das Antas e 42,1 $\mathrm{Km}^{2}$ na REBIO União. Já para $C$. nigritus a densidade populacional foi estimada para $66,2 \mathrm{ind} / \mathrm{Km}^{2}$ na $\mathrm{REBIO}$ de Poço das Antas e 34,0 ind/ $\mathrm{Km}^{2}$ para a REBIO União. Dasypus novemcinctus apresentou densidade estimada para 18,9 ind $/ \mathrm{Km}^{2}$ na REBIO de Poço das Antas e 11,0 ind/ $\mathrm{Km}^{2}$ na REBIO União (Tab. V). Cebus nigritus mostrou ter tamanho populacional maior na REBIO de Poço das Antas, enquanto que a população de $A$. guariba apresentou-se maior na REBIO União (Tab. V).

\section{DISCUSSÃO}

A variação na abundância e na densidade das espécies em diferentes locais é atribuída principalmente às diferenças na composição e na estrutura do habitat (August, 1983; Scharwzkopf \& Rylands, 1989; Peres, 1997), disponibilidade de recursos (STEVENSON, 2001), competição por recursos (TERBORGH, 1983; EsTRADA \& Coates-Estrada, 1985), presença de predadores (StANFORD, 1995; Wright et al., 1998), plasticidade ecológica (RobInson \& REDFORd, 1986; PERES, 1997; SteVEnson, 2001), caça ilegal e fragmentação florestal (Peres, 1997; Cullen et al., 2000; Chiarello, 2000b).

Os resultados deste trabalho indicam que a caça, apesar de proibida pela lei federal, continua sendo praticada nas áreas de estudo. Porém, caçadores têm agido de forma profissional principalmente na REBIO União, onde grandes acampamentos foram flagrados pela equipe de pesquisadores no campo. Este fato pode ser explicado devido à carência de fiscais nesta Unidade de Conservação. A REBIO de Poço das Antas possui funcionários que trabalham na fiscalização, porém o seu número é reduzido, e estes são acionados ocasionalmente pelo IBAMA da REBIO União.

As espécies cinegéticas mais abundantes foram os primatas de grande porte ( $A$. guariba e $C$. nigritus), o que pode indicar que estas espécies não são preferencialmente caçadas na região. As densidades estimadas de $C$. nigritus em outros fragmentos de Mata Atlântica foram revisadas (Tab.VI) sendo que a densidade para a REBIO Poço das Antas foi a maior reportada.

Em contrapartida, $P$. tajacu e D. leporina foram os mamíferos menos abundantes nas duas Unidades de

Tabela V. Densidade (indivíduos $/ \mathrm{km}^{2}$ ), coeficiente de variação, ESW (metros), abundância (encontros/10 Km) e tamanho populacional de mamíferos cinegéticos na Reserva Biológica de Poço das Antas e na Reserva Biológica União, Rio de Janeiro.

\begin{tabular}{llcrrcc}
\hline Local & \multicolumn{1}{c}{ Espécie } & Densidade $\left(\mathrm{ind} / \mathrm{Km}^{2}\right)$ & CV & ESW (m) & $\begin{array}{c}\text { Abundância } \\
\text { (enc./10 Km) }\end{array}$ & $\begin{array}{c}\text { Tamanho } \\
\text { populacional }\end{array}$ \\
\hline Poço das Antas & Alouatta guariba & $44,1(38,3-48,7)$ & 0,13 & 11,30 & 0,72 & 945,90 \\
& Cebus nigritus & $66,2(55,0-96,1)$ & 0,18 & 10,46 & 1,15 & $1.419,0$ \\
& Pecari tajacu & $0,04(0,02-0,06)$ & 0,18 & 11,14 & 0,30 & - \\
& Dasyprocta leporina & $0,13(0,09-0,15)$ & 0,19 & 7,26 & 0,30 & - \\
& Dasypus novemcinctus & $18,9(15,1-25,8)$ & 0,16 & 6,10 & 1,18 & 405,40 \\
União & Dasypus septemcinctus & $0,22(0,15-028)$ & 0,17 & 11,10 & 0,27 & - \\
& Alouatta guariba & $42,1(36,7-47,5)$ & 0,15 & 10,05 & 1,11 & $1.353,50$ \\
& Cebus nigritus & $34,0(29,8-39,4)$ & 0,13 & 14,30 & 0,44 & $1.093,0$ \\
& Pecari tajacu & $0,07(0,04-0,11)$ & 0,19 & 5,14 & 0,30 & - \\
& Dasyprocta leporina & $0,09(0,06-0,10)$ & 0,22 & 5,63 & 0,22 & - \\
& Dasypus novemcinctus & $11,0(0,78-15,4)$ & 0,17 & 6,11 & 0,83 & 350,50 \\
& Dasypus septemcinctus & $0,20(0,13-0,27)$ & 0,18 & 5,17 & 0,38 & - \\
\hline
\end{tabular}


Tabela VI. Densidade populacional de bugio (A. guariba), macaco-prego (C. nigritus) e cutia (D. leporina) em diferentes fragmentos de Mata Atlântica (1, Pinto et al., 1993; 2, Peres, 1997; 3, Chiarello \& Melo, 2001; 4, Cullen \& valladares-Padua, 1997; 5, Mendes, 1989; 6, Cullen et al., 1999; 7, Bernardo, 2004).

\begin{tabular}{|c|c|c|c|c|}
\hline Espécies & Local/Estado & Área $\left(\mathrm{Km}^{2}\right)$ & Densidade (ind./Km²) & Referência \\
\hline \multirow{8}{*}{ A. guariba } & Parque Estadual Ilha do Cardoso (SP) & 111,00 & 8,90 & 7 \\
\hline & Reserva Biol. A. Rushi (ES) & 40,00 & 10,10 & 1 \\
\hline & Fazenda Barreiro Rico (SP) & 32,60 & $14-38$ & 1 \\
\hline & Reserva Biol. União (RJ) & 31,26 & 42,10 & Presente estudo \\
\hline & Reserva Biol. de Poço das Antas (RJ) & 55,00 & 44,10 & Presente estudo \\
\hline & Floresta Nac. de 3 Barras (SC) & 44,60 & 64,00 & 2 \\
\hline & Parque Estadual da Cantareira (SP) & 56,50 & 80,90 & 1 \\
\hline & Estação Biol. Caratinga (MG) & 8,80 & 117,00 & 5 \\
\hline \multirow{7}{*}{ C. nigritus } & Reserva Biol. A. Rushi (ES) & 40,00 & 10,20 & 1 \\
\hline & Reserva Biol. do Córrego do Veado (ES) & 24,00 & 10,40 & 3 \\
\hline & Reserva Caetetus (SP) & 21,80 & 17,60 & 4 \\
\hline & Reserva Biol. de Linhares (ES) & 218,00 & 24,30 & 3 \\
\hline & Fazenda Barreiro Rico (SP) & 32,60 & $25-50$ & 1 \\
\hline & Reserva Biol. União (RJ) & 31,26 & 34,00 & Presente estudo \\
\hline & Reserva Biol. de Poço das Antas (RJ) & 55,00 & 66,20 & Presente estudo \\
\hline \multirow{5}{*}{ D. leporina } & Reserva Biol. União (RJ) & 31,26 & 0,09 & Presente estudo \\
\hline & Reserva Biol. de Poço das Antas (RJ) & 55,00 & 0,13 & Presente estudo \\
\hline & Fazenda Mosquito (SP) & 21,00 & 0,39 & 6 \\
\hline & Fazenda Rio Claro (SP) & 17,00 & 0,91 & 6 \\
\hline & Parque Estadual Ilha do Cardoso (SP) & 111,00 & 3,40 & 7 \\
\hline
\end{tabular}

Conservação estudadas. Dasyprocta leporina apresentou baixa densidade na área de estudo, em comparação com outros remanescentes florestais de tamanho similar (Tab. VI). Cabe destacar que este mamífero possui alto valor cinegético (REDFORD \& ROBINSON, 1987) e sua extinção local pode trazer consequiências prejudiciais para diversas espécies arbóreas, já que esses roedores dispersam sementes enterrando-as no chão da mata (WUNDERLE JR, 1997; SILVA \& TABARELLI, 2001; GALETTI et al., 2006), favorecendo a germinação e a taxa de sobrevivência de sementes, além de influenciar na disponibilidade de alimento para outros consumidores (LAMBERT \& GARBER, 1998).

O tamanho populacional é um dos principais fatores que determinam o risco de extinção ou a persistência de espécies numa determinada área, sendo que as populações pequenas têm maior probabilidade de extinção quando comparadas às populações maiores (BRITO \& FERNANDEZ, 2000; REED et al., 2003). No caso da REBIO de Poço das Antas, há um grande isolamento deste fragmento, que atua como barreira geográfica para as espécies estudadas, dificultando o fluxo gênico entre as populações.

Para algumas espécies de mamíferos, somente áreas de mata com mais de 20.000 ha sustentam populações grandes o suficiente para escapar da extinção por um longo tempo (ChiARELlo, 2000a). No entanto, apenas 20\% de todas as Reservas Biológicas da Mata Atlântica brasileira são maiores que 20.000 ha (IBGE, 1993). O maior tamanho populacional estimado para A. guariba, $C$. nigritus e D. novemcinctus nas duas Unidades de Conservação não foi superior a 1.500 indivíduos (Tab. V). A população mínima viável estimada por (REED et al., 2003) para 102 espécies de vertebrados foi de cerca de 7.000 indivíduos.

As espécies mais raras consideradas neste estudo foram $H$. hydrochaeris, T. terrestris, M. americana e $T$. pecari. Espécies de grande porte, que possuem baixas taxas reprodutivas e maior longevidade são mais vulneráveis do que as espécies de vida curta e com maior capacidade reprodutiva. Assim, tais espécies como as antas e os porcos- do-mato são considerados mais vulneráveis à pressão de caça do que as demais (BODMER et al., 1997).

\section{CONCLUSÕES}

Espécies cinegéticas ainda existem nas Reservas Biológicas de Poço das Antas e União, mas a maioria das populações é constituída de poucos indivíduos. Estas pequenas populações são mais sensíveis à extinção local e podem não ser viáveis a longo prazo.

Os dados sobre parâmetros populacionais de espécies de alto valor cinegéticos são preocupantes e indicam que a existência da prática da caça na região está influenciando na presença destas espécies. A caça, apesar de proibida pela lei federal, continua sendo praticada e as Unidades de Conservação podem não estar atuando efetivamente na proteção da fauna. Um trabalho de educação ambiental somado a uma melhoria do sistema de fiscalização nas Unidades de Conservação seriam fatores importantes para conservação de espécies cinegéticas na região.

Agradecimentos. À Idea Wild e a Associação Mico-LeãoDourado/Lion Tamarin Brazilian Found (AMLD/LTBF) pelo financiamento do projeto. A Universidade Estadual do Norte Fluminense/ Laboratório de Ciências Ambientais (UENF/LCA) pelo apoio logístico. Ao técnico de campo Helzenil Cordeiro (Nil) e ao MSc. Marcio M. de Morais pela ajuda na coleta dos dados no campo. Ao Dr. Alex Pury, do Setor de Geoprocessamento (UENF/ LCA), por ter elaborado e cedido o mapa. A MSc. Daniela Teodoro Sampaio e a Renata Ribeiro Gomes pela revisão do manuscrito.

\section{REFERÊNCIAS BIBLIOGRÁFICAS}

AMLd. Associação Mico-Leão-Dourado. 2003. Relatório Institucional. Oliveira, P. P. \& Rambaldi, D. M. Silva Jardim, RJ. 54p.

August, P. V. 1983. The role of habitat complexity and heterogeneity in structuring tropical mammal communities. Ecology 64:1495-1507.

Bodmer, R. E.; Eisenberg, J. F. \& Rerford, K. H. 1997. Hunting and the likelihood of extinction of Amazonian mammals. Conservation Biology 11:460-466. 
Brito, D. \& Fernandez, F. A. S. 2000. Dealing with extinction is forever: understanding the risks faced by small populations. Journal of the Brazilian Association for the Advancement of Science, 52(3):161-170.

Brito, D.; Oliveira, L. \& Mello, M. A. R. 2004. An overview of mammalian conservation at Poço das Antas Biological Reserve, southeastern Brazil. Journal of Nature Conservation 12:219-228.

Buckland S. T.; Anderson, D. R.; Burnham K. P. \& Laake, J. L. 1993. Distance Sampling, Estimating Abundance of Biological Populations. London. Chapman \& Hall. 432p.

Buckland S. T.; Anderson, D. R.; Burnham K. P.; Laake, J. L. \& BoEchers, D. L. 2001. Introduction to distance sampling: estimating abundance of biological populations. Oxford, Oxford University. 568p.

Chiarello, A. G. 1999. Effects of fragmentation of the Atlantic forest on mammal communities in South-eastern Brazil. Biological Conservation 89:71-82. 2000a. Density and population size of mammals in remnants of Brazilian Atlantic forest. Conservation Biology 14:1649-1657. . 2000b. Influência da caça ilegal sobre os mamíferos e aves das matas de tabuleiro do norte do estado do Espírito Santo. Boletim do Museu de Biologia Mello Leitão 11/12:229-247.

Chiarello, A. G. \& Melo, F. R. 2001. Primate population densities and sizes in Atlantic forest remnants of Northern Espírito Santo, Brazil. International Journal of Primatology 22(3):379-394

Cullen, L. \& Valladares-Pádua, C. 1997. Métodos para estudos de ecologia, manejo e conservação de primatas na natureza. In Valladares-Padua, C.; Bodmer, R. \& Cullen, L. eds. Manejo e conservação da vida selvagem no Brasil. Brasília, Sociedade Civil Mamirauá. 254p.

Cullen, L.; Bodmer, R. E. \& Valladares-Pádua, C. 1999. Caça e biodiversidade nos fragmentos florestais da Mata Atlântica, São Paulo, Brasil. In: FAnG, T. G.; Montenegro, O. L. \& Bodmer, R. eds. Manejo y conservación de fauna silvestre em América Latina. Flórida, University of Florida.

Cullen, L.; Valladares-PÁduA, C. \& Bodmer, R. E. 2000. Effects of hunting in habitat fragments of the Atlantic forest, Brazil. Biological Conservation 95:49-65.

Cullen, L.; Rudran, R. \& Valladares-Padua, C. 2003. Métodos de estudos em biologia da conservação e manejo da vida silvestre. Curitiba, Editora UFPR. 665p.

Estrada, A. \& Coates-Estrada, R. A. 1985. A preliminary study of resource overlap between howling monkeys (Alouatta palliata) and other arboreal mammals in the tropical rainforest of Los Tuxtlas, Mexico. American Journal of Primatology 9:27-37.

Ferrari, S. F. 2002. Multiple transects or multiple walks? A response to Magnusson (2001). Neotropical Primates 10(3): $131-132$

Galetti, M.; Martuscelli, P.; Olmos, F. \& Aleixo, A. 1997. Ecology and conservation of the jacutinga Pipile jacutinga in the Atlantic forest of Brazil. Conservation Biology 82:31-39.

Galetti, M.; Donatti, C. I.; Pires, A. S.; Guimarães, P. R. \& Jordano, P. 2006. Seed survival and dispersal of an endemic Atlantic forest palm: the combined effects to defaunation and forest fragmentation. Botanical Journal of the Linnean Society, 151:141-149.

IBGE. Instituto Brasileiro de Geografia e Estatística. 1993. Mapa da vegetação do Brasil. Rio de Janeiro, Fundação Instituto Brasileiro de Geografia e Estatística.

LAmbert, J. E. \& GARBER, P. A. 1998. Evolutionary and ecological implications of primate seed dispersal. American Journal of Primatology 45(1):9-28.

LANE, F. 1990. A hunt for monos (Brachyteles arachnoides) in the footthills of the Serra da Paranapiacaba, São Paulo, Brasil. Primate Conservation 11:23-25.

Mendes, S. L. 1989. Estudo ecológico de Alouatta fusca (Primates: Cebidae) na Estação Ecológica de Caratinga, MG. Revista Nordestina de Biologia 6(2):71-104.

Mittermeier, R. A.; Myers, N.; Thomsen, J. B.; Fonseca, G. A. \& Olivieri, S. 1998. Biodiversity hotspots and major tropical wilderness area approaches to setting conservation priorities. Conservation Biology 12:516-520.

Myers, N.; Mittermeier, R. A.; Mittermeier, C. G.; Fonseca, G. A. B \& Kent, J. 2000. Biodiversity hotspots for conservation priorities. Nature 403:853-858.
Olmos, F.; Alburoueque, J. L.; Galetti, M.; Milano, M. S.; Camara, I. G.; Coimbra-Filho, A. F.; Pacheco, J. F.; Bauer, C.; Pena, C. G.; Freitas, T. R. O.; Pizo, M. A. \& Aleixo, A. 2002. A correlação política e biodiversidade: a ameaça das populações tradicionais à Mata Atlântica. In: Albuquerque, J. L. B.; CAndido, J. F.; Straube, F. C. \& Roods, E. A. eds. Ornitologia e conservação: da ciência às estratégias. Tubarão, UNISUL. 150p.

Peres, C. A. 1997. Effects of habitat quality and hunting pressure on arboreal folivore densities in neotropical forests: a case study of howler monkeys (Alouatta spp.). Folia Primatologica 68:199-222.

Peres, C. A. 2000. Evaluating the impact and sustainability of subsistence hunting at multiple Amazonian forest sites. In: Robinson, J. G. \& BennetT, E. L. eds. Hunting for Sustainability in Tropical Forests. New York, Columbia University. p.83-115.

Pinto, L. P. S.; Costa, C. M. R.; Strier, K. B. \& Fonseca, G. A. B 1993. Habitat, density and group size of primates in a Brazilian Tropical Forest. Folia Primatologica 61:135-143.

Pires, A. S. \& Fernandez, F. A. S. 1999. Use of space by the marsupial Micoureus demerarae in small Atlantic Forest fragments in south-eastern Brazil. Journal of Tropical Ecology 15:279-290.

Pires, A. S.; Lira, P. K.; Fernandez, F. A. S.; Schittini, G. M. \& Oliveira, L. C. 2002. Frequency of movements of small mammals among Atlantic Coastal Forest fragments in Brazil. Biological Conservation 108:229-237.

Redford, K. H. \& Robinson, J. G. 1987. The game of choice: patterns of indian and colonist hunting in the neotropics. American Anthropolist 89:650-667.

REDFord, K. H. 1992. The empty forest. Bioscience 42:412-422.

Reed, D. H.; O'Grady, J. L.; Brook, B. W.; Ballou, J. D. \& FrankHAM, R. 2003. Estimates of minimum viable population sizes for vertebrates and factors influencing those estimates. Biological Conservation 113:23-34

Robinson, J. G. \& Redford, K. H. 1986. Body size, diet and population density of neotropical forest mammals. American Naturalist 128:665-680.

Rocha, C. F. D.; Bergallo, H. G.; Alves, M. A. S. \& Sluys, M. V. 2003. A biodiversidade nos grandes remanescentes florestais do Estado do Rio de Janeiro e nas restingas da Mata Atlântica. São Carlos, Editora RIMA. 134p.

SchwarzKopfD, L. \& Rylands, A. B. 1989. Primates species richness in relation to habitat structure in Amazonian rainforest fragments. Biological Conservation 48:1-12.

SEMA. Secretaria de Estado de Meio Ambiente e Desenvolvimento Sustentável, Governo do Estado do Rio de Janeiro. 2001. Atlas das Unidades de Conservação da Natureza do Estado do Rio de Janeiro. Rio de Janeiro, Metalivros. 48p.

Silva, M. G. \& TABARElli, M. 2001. Seed dispersal, plant recruitment and spatial distribution of Bactris acanthocarpa Martius (Arecaceae) in a remnant of atlantic Forest in northeast Brazil. Acta Oecologia 22:259-268

SOS MATA ATLÂNTICA \& INPE. 1993. Evolução dos remanescentes florestais e ecossistemas associados ao domínio da Mata Atlântica no período de 1985-1990. São Paulo, SOS Mata Atlântica e Instituto de Pesquisas Espaciais. 199p.

StAnFord, C. B. 1995. The influence of chimpanzee predation on group size and antipredator behaviour in red colobus monkeys. Animal Behaviour 49:577-587.

Stevenson, P. R. 2001. The relationship between fruit production and primate abundance in neotropical communities. Biological Journal of the Linnean Society 72:161-178.

TAniZAKI-FonsecA, K. \& Moulton, T. P. 2000. A fragmentação da Mata Atlântica no Estado do Rio de Janeiro e a perda de biodiversidade. In: Bergallo, H. G.; Rocha, C. F. D.; Alves, M. A. S. \& SLuYs, M. V. eds. A fauna ameaçada de extinção do Estado do Rio de Janeiro. Rio de Janeiro, Editora UERJ. p.23-35.

TERBORGH, J. 1983. Five new world primates: a study in comparative ecology. Princeton, Princeton University. 260p.

WRIGHT, S. J. 1998. Impact of predation risk on the behaviour of Propithecus diadema ewardsi in the rainforest of Madagascar. Behaviour 135:485-512.

Wunderle JR., J. M. 1997. The role of animal seed dispersal in accelerating native forest regeneration on degraded tropical lands. Forestry Ecology and Management 99:223-235. 